\title{
Flickr1024: A Large-Scale Dataset for Stereo Image Super-Resolution
}

\author{
Yingqian Wang ${ }^{1}$, Longguang Wang ${ }^{1}$, Jungang Yang ${ }^{1 *}$, Wei $\mathrm{An}^{1}$, and Yulan Guo ${ }^{1}$ \\ ${ }^{1}$ College of Electronic Science and Technology, National University of Defense Technology, China \\ \{wangyingqian16, yangjungang\}@nudt.edu.cn
}

\begin{abstract}
With the popularity of dual cameras in recently released smart phones, a growing number of super-resolution (SR) methods have been proposed to enhance the resolution of stereo image pairs. However, the lack of high-quality stereo datasets has limited the research in this area. To facilitate the training and evaluation of novel stereo SR algorithms, in this paper, we present a large-scale stereo dataset named Flickr1024, which contains 1024 pairs of high-quality images and covers diverse scenarios. We first introduce the data acquisition and processing pipeline, and then compare several popular stereo datasets. Finally, we conduct crossdataset experiments to investigate the potential benefits introduced by our dataset. Experimental results show that, as compared to the KITTI and Middlebury datasets, our Flickr1024 dataset can help to handle the over-fitting problem and significantly improves the performance of stereo SR methods. The Flickr1024 dataset is available online at: https://yingqianwang.github.io/Flickr1024.
\end{abstract}

\section{Introduction}

With recent advances in camera miniaturization, dual cameras are commonly adopted in commercial mobile phones. Using the complementary information provided by binocular systems, the resolution of image pairs can be enhanced by stereo super-resolution (SR) methods $[2,6,17]$. Nowadays, many top-performing SR methods $[6,16,17,19]$ are built upon deep neural networks. These data-driven SR methods can be enormously benefited from large-scale high-quality datasets such as DIV2K [1] and Vimeo-90K [18].

In the area of stereo vision, several datasets are currently available. The KITTI stereo datasets $[4,10]$ are mainly developed for autonomous driving. All images in the KITTI2012 [4] and KITTI2015 [10] datasets are captured by two video cameras mounted on the top of a car. The scenes in the KITTI datasets only include roads or highways from driving perspectives. Groundtruth disparity is provided for the training of stereo matching and vi-

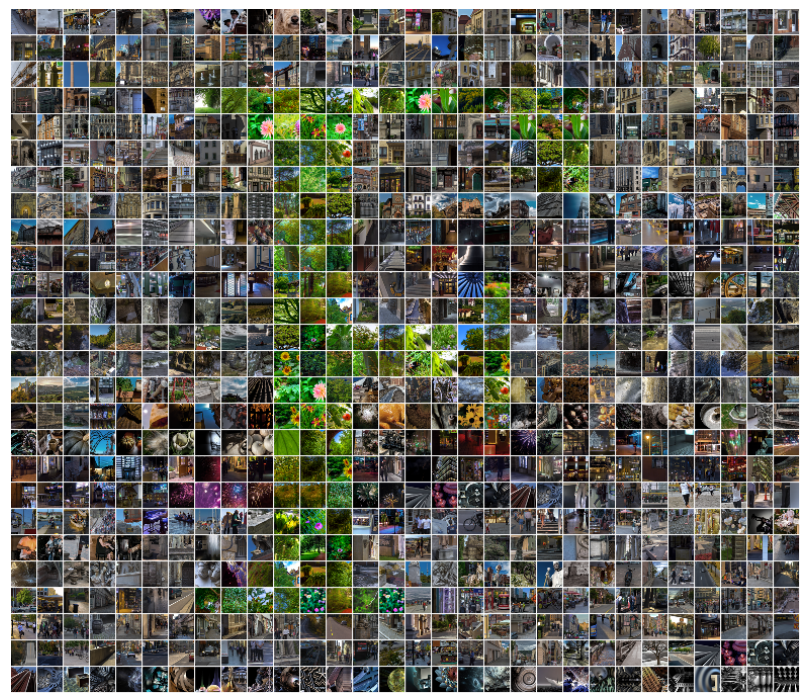

Figure 1: The Flickr1024 dataset.

sual odometry. The Middlebury stereo dataset consists of a series of sub-datasets introduced in 2003 [14], 2005 [13], 2006 [5], and 2014 [12]. The Middlebury dataset is acquired in the laboratory, its scenes only cover close-shots of different objects. Note that, 55 of its 65 image pairs are provided with groundtruth disparity for stereo matching. The ETH3D stereo dataset is a part of the ETH3D benchmark [15]. Groundtruth depth is provided for visual odometry and $3 \mathrm{D}$ reconstruction. Note that, images in the ETH3D dataset are of gray scale, of low resolution, and with limited scenarios.

Since the task of stereo vision can vary significantly (e.g., stereo matching [8], stereo segmentation [7]), existing stereo datasets are unsuitable for stereo SR due to the insufficient number of images and limited types of scenarios. To design, train, and evaluate novel stereo SR methods, a largescale and high-quality stereo dataset with diverse scenarios is highly needed. In this paper, we present a new Flickr1024 dataset (see Fig. 1) for stereo SR. In summary, the contribu- 


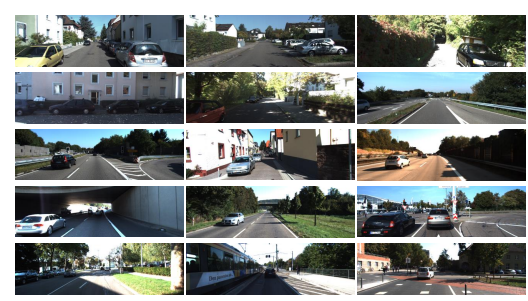

The KITTI Dataset

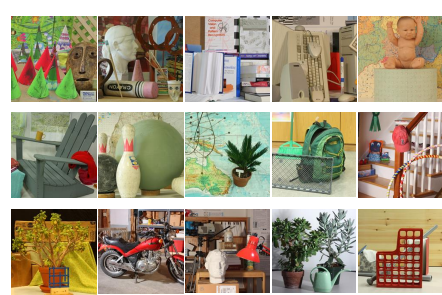

The Middlebury Dataset

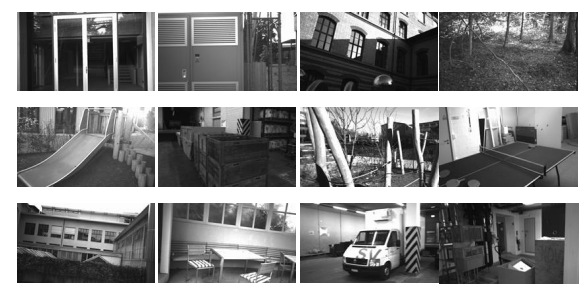

The ETH3D Dataset
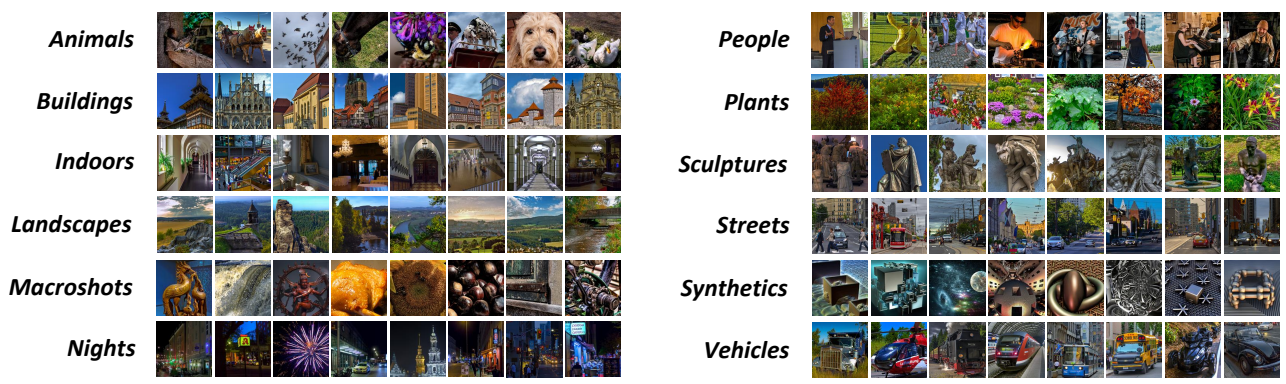

The Flickr1024 Dataset

Figure 2: Example images sampled from several popular stereo datasets: KITTI [4,10], Middlebury [5, 12-14], ETH3D [15], and Flickr1024. It can be observed that our Flickr1024 dataset covers significantly more diverse scenarios as compared to the existing stereo datasets.

tions of our dataset can be listed as follows:

- It is the largest dataset for stereo SR to date, which contains 1024 high-quality image pairs and covers diverse scenarios.

- The scenarios covered by our dataset are highly consistent with real cases in daily photography (see Fig. 2). Consequently, algorithms developed on the Flickr1024 dataset can be easily adopted in real-world applications such as mobile phones.

- Experimental results demonstrate that our dataset can help to address the over-fitting problem and significantly improve the performance of different stereo SR methods. That is, our dataset can benefit both industrial and research communities in stereo SR.

The Flickr1024 dataset was initially introduced in our previous conference paper [17] and used as the augmented training data for our PASSRnet algorithm. However, in our preliminary work, both details and effectiveness of this dataset have not been investigated. In this paper, we deeply investigate the Flickr1024 dataset and make several additional contributions, which can be summarized as follows:

- We provide more details in data acquisition and processing. The pipelines and processing methods used in this paper can help the community to develop new datasets for their own research.
- We comprehensively compare the Flickr1024 dataset to several existing stereo datasets $[4,5,10,12-15]$ using different objective metrics. Evaluation results have demonstrated the superiority of our dataset.

- We conduct cross-dataset experiment to study the performance gains introduced by the Flickr1024 dataset. Experimental results have demonstrated the effectiveness of our dataset in performance promotion and overfitting elimination.

\section{Data Acquisition and Processing}

To generate the Flickr1024 dataset, we manually collected 1024 RGB stereo photographs from albums on Flickr ${ }^{1}$ with the permissions of photograph owners. Since all images collected from Flickr are in cross-eye pattern for $3 \mathrm{D}$ visualization, their optical axes should be corrected to be parallel. As shown in Fig. 3, the processing pipeline can be summarized as follows:

1. We cut each cross-eye photograph into a stereo image pair. Note that, to transform a cross-eye photograph into an image pair with parallel optical axis, the left and right images in the stereo image pair need to be exchanged.

\footnotetext{
${ }^{1}$ https://www.flickr.com/
} 
Table 1: Main characteristics of several popular stereo datasets. Both average value and standard deviation are reported. Among all the compared datasets, the Flickr1024 dataset achieves promising scores in image pairs, resolution, and perceptual image quality.

\begin{tabular}{|l|cccccc|}
\hline Datasets & Image Pairs & Resolution $(\uparrow)$ & Entropy $(\uparrow)$ & BRISQE $(\downarrow)[11]$ & SR-metric $(\uparrow)[9]$ & ENIQA $(\downarrow)[3]$ \\
\hline KITTI2012 [4] & 389 & $0.46( \pm 0.00) \mathrm{Mpx}$ & $7.12( \pm 0.30)$ & $\mathbf{1 7 . 4 9}( \pm 6.56)$ & $\mathbf{7 . 1 5}( \pm 0.63)$ & $\underline{0.097}( \pm 0.028)$ \\
KITTI2015 [10] & $\underline{400}$ & $0.47( \pm 0.00) \mathrm{Mpx}$ & $7.06( \pm 0.00)$ & $23.79( \pm 5.81)$ & $7.06( \pm 0.51)$ & $0.169( \pm 0.030)$ \\
Middlebury [5,12-14] & 65 & $\mathbf{3 . 5 9}( \pm 2.06) \mathrm{Mpx}$ & $\mathbf{7 . 5 5}( \pm 0.20)$ & $26.85( \pm 13.30)$ & $6.01( \pm 1.08)$ & $0.270( \pm 0.120)$ \\
ETH3D [15] & 47 & $0.38( \pm 0.08) \mathrm{Mpx}$ & $\underline{7.24}( \pm 0.43)$ & $27.95( \pm 12.06)$ & $5.99( \pm 1.52)$ & $0.195( \pm 0.073)$ \\
Flickr1024 & $\mathbf{1 0 2 4}$ & $\underline{0.73}( \pm 0.33) \mathrm{Mpx}$ & $7.23( \pm 0.64)$ & $\underline{19.40}( \pm 13.77)$ & $\underline{7.12}( \pm 0.67)$ & $\mathbf{0 . 0 6 5}( \pm 0.073)$ \\
Flickr1024 (Train) & 800 & $0.74( \pm 0.34) \mathrm{Mpx}$ & $7.23( \pm 0.65)$ & $19.10( \pm 13.69)$ & $7.12( \pm 0.66)$ & $0.063( \pm 0.074)$ \\
Flickr1024 (Validation) & 112 & $0.72( \pm 0.23) \mathrm{Mpx}$ & $7.26( \pm 0.54)$ & $20.03( \pm 12.54)$ & $7.13( \pm 0.70)$ & $0.074( \pm 0.084)$ \\
Flickr1024 (Test) & 112 & $0.72( \pm 0.32) \mathrm{Mpx}$ & $7.22( \pm 0.60)$ & $20.97( \pm 15.40)$ & $7.12( \pm 0.67)$ & $0.076( \pm 0.087)$ \\
\hline
\end{tabular}

Note: Mpx denotes megapixels per image. The best scores are in bold and the second best scores are underlined.

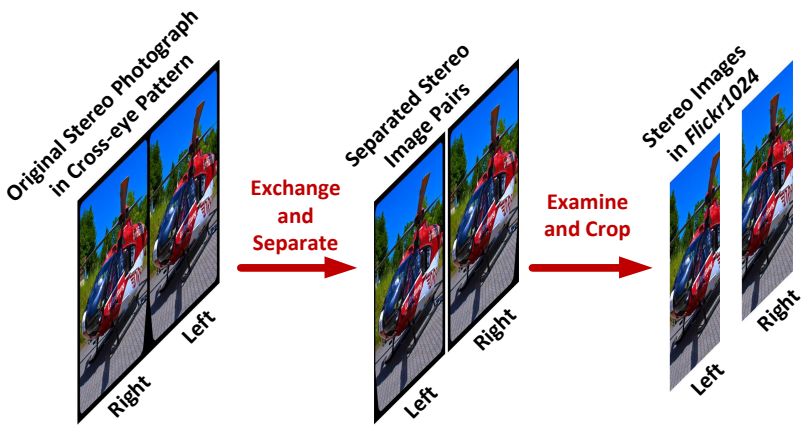

Figure 3: The processing pipeline to generate the Flickr1024 dataset.

2. We check each pair of stereo images to ensure that they are vertically rectified (i.e., image pairs has horizontal disparities only). In practice, most image pairs have already been calibrated in vertical direction by the photo owners. For these images without vertical calibration, we simply discard them from our dataset.

3. We crop the left and right images to remove black (or white) margins and to make zero disparity corresponding to infinite depth. Note that, regions with infinite depth are unavailable for close-shot images. We therefore, crop these image pairs to ensure that the minimum disparity is larger than a certain value (set to 40 pixels in our dataset).

Finally, we randomly split our dataset to generate 800 training image pairs, 112 validation image pairs, and 112 test image pairs.

\section{Comparisons to Existing Datasets}

In this section, statistical comparisons are performed to demonstrate the superiority of the Flickr1024 dataset.
The main characteristics of the Flickr1024 dataset and four existing stereo datasets [4, 5, 10, 12-15] are listed in Table 1. Following [1], we use entropy to measure the amount of information included in each dataset, and use three no-reference image quality assessment (NRIQA) metrics to assess the perceptual image quality, including blind/referenceless image spatial quality evaluator (BRISQE) [11], SR-metric [9], and entropy-based image quality assessment (ENIQA) [3]. For image quality assessment, these NRIQA metrics are superior to many fullreferenced measures (e.g., PSNR, RMSE, and SSIM), and highly correlated to human perception [9]. For all the NRIQA metrics presented in this paper, we run the codes provided by their authors under their original models and default settings. Note that, small values of BRISQE [11] and ENIQA [3], and large values of SR-metric [9] represent high image quality.

As listed in Table 1, the Flickr1024 dataset is larger than other datasets by at least 2.5 times. Besides, the image resolution of the Flickr1024 dataset also outperforms that of the KITTI2012, KITTI2015, and ETH3D datasets. Although the Middlebury dataset has the highest image resolution, the number of image pairs in this dataset is limited. The entropy values of all datasets are comparable, while the entropy of the KITTI datasets is relatively low. That is, the diversity of images in the KITTI datasets is smaller than that of other datasets. For perceptual image quality assessment, both the Flickr1024 and the KITTI2012 datasets achieve promising scores. Specifically, the Flickr1024 dataset has the highest ENIQA score, and the second highest BRISQE and SRmetric scores. Since these metrics are influenced by the brightness and textures of tested images, the Flickr1024 dataset has higher standard deviations than existing datasets due to its diverse scenarios. These assessments indicate that images in Flickr1024 are relatively high in perceptual quality and suitable for stereo SR. 
Table 2: PSNR and SSIM values achieved by StereoSR [6] for $4 \times$ SR with 60 training epochs.

\begin{tabular}{|c|c|c|c|c|}
\hline Dataset & KITTI2015 (Test) & Middlebury (Test) & Flickr1024 (Test) & ETH3D (Test) \\
\hline KITTI2015 (Train) & $24.28 / 0.741$ & $26.27 / 0.749$ & $21.77 / 0.617$ & $29.63 / 0.831$ \\
\hline Middlebury (Train) & $23.64 / 0.743$ & $26.62 / 0.773$ & $21.64 / 0.646$ & $28.66 / 0.843$ \\
\hline Flickr1024 (Train) & $\mathbf{2 5 . 0 8 / 0 . 7 7 9}$ & $\mathbf{2 7 . 8 5 / 0 . 8 0 7}$ & $\mathbf{2 2 . 6 4 / 0 . 6 9 2}$ & $\mathbf{3 0 . 5 5 / 0 . 8 6 0}$ \\
\hline
\end{tabular}

Table 3: PSNR and SSIM values achieved by PASSRnet [17] for $4 \times$ SR with 80 training epochs.

\begin{tabular}{|c|c|c|c|c|}
\hline Dataset & KITTI2015 (Test) & Middlebury (Test) & Flickr1024 (Test) & ETH3D (Test) \\
\hline KITTI2015 (Train) & $23.13 / 0.703$ & $25.42 / 0.762$ & $21.31 / 0.600$ & $26.95 / 0.789$ \\
\hline Middlebury (Train) & $25.18 / 0.774$ & $28.08 / 0.853$ & $22.54 / 0.676$ & $31.39 / 0.864$ \\
\hline Flickr1024 (Train) & $\mathbf{2 5 . 6 2 / 0 . 7 9 1}$ & $\mathbf{2 8 . 6 9 / 0 . 8 7 3}$ & $\mathbf{2 3 . 2 5 / 0 . 7 1 8}$ & $\mathbf{3 1 . 9 4 / 0 . 8 7 7}$ \\
\hline
\end{tabular}

It is also notable that, comparable scores of these metrics can be achieved on three different subsets (i.e., training set, validation set, and test set) of the Flickr1024 dataset, as shown in Table 1. That means, a good balance is achieved with random partition, and the bias between the training and the test process is relatively small.

\section{Cross-Dataset Evaluation}

To investigate the potential benefits of a large-scale dataset to the performance improvement of learning-based stereo SR methods, experimental results are provided in this section. Besides, a cross-dataset evaluation is performed to fully demonstrate the superiority of the Flickr1024 dataset.

\subsection{Implementation Details}

We use two state-of-the-art stereo SR methods (i.e., StereoSR [6] and PASSRnet [17]) in this experiment. These two methods are first trained on the KITTI2015, Middlebury, and Flickr1024 datasets, and then tested on the above three datasets and the ETH3D dataset. For simplification, only $4 \times$ SR models are investigated. That is, stereo image pairs are first down-sampled by a factor of 4 , and then super-resolved to their original resolutions. We compare the reconstructed image with the original image, and use PSNR and SSIM for performance evaluation.

We used the codes of StereoSR [6] and PASSRnet [17] released by their authors. Since the StereoSR model trained on the Middlebury dataset is available, we directly use this model in our experiment. For the other 5 unavailable models, we retrain these two SR methods.

\subsection{Results}

Tables 2 and 3 present the results of StereoSR and PASSRnet trained with fixed training epochs. We can observe that both algorithms trained on the Flickr1024 dataset achieve the highest PSNR and SSIM values on all of the test sets as compared to those trained on the KITTI2O15 and Middlebury datasets. Specifically, the Flickr1024 dataset out- performs the second best datasets (KITTI2015 in Table 2 and Middlebury in Table 3) by 1.04 and 0.58 in average PSNR values, respectively. These results demonstrate that the Flickr1024 dataset can help to significantly improve the performance of stereo SR algorithms.

Moreover, we train PASSRnet [17] with different training epochs, and further investigate the variation of PSNR and SSIM values. The results are shown in Fig. 4, where each sub-figure illustrates the performance tested on a specific dataset. We can observe that the algorithm trained on the Flickr1024 dataset achieves the highest PSNR and SSIM values with any number of training epochs. For the models trained on the KITTI2015 dataset, their PSNR and SSIM curves suffer a downward trend. In contrast, the models trained on the Flickr1024 dataset can achieve a gradually improved performance with an increasing number of training epochs. These results demonstrate that, by using our dataset, a reasonable convergence can be steadily achieved and the over-fitting issue can be well addressed.

\section{Conclusion}

In this paper, we introduce Flickr1024, a large-scale dataset for stereo SR. The Flickr1024 dataset consists 1024 high-quality images and covers diverse scenarios. Both statistical comparisons and cross-dataset experiments demonstrate the effectiveness of our dataset. That is, the Flickr1024 dataset can be used to improve the performance of learning-based stereo SR methods. The Flickr1024 dataset can also help to boost the reseach in stereo superresolution.

\section{Acknowledgment}

The authors would like to thank Sascha Becher and Tom Bentz for the approval of using their cross-eye stereo photographs on Flickr. 

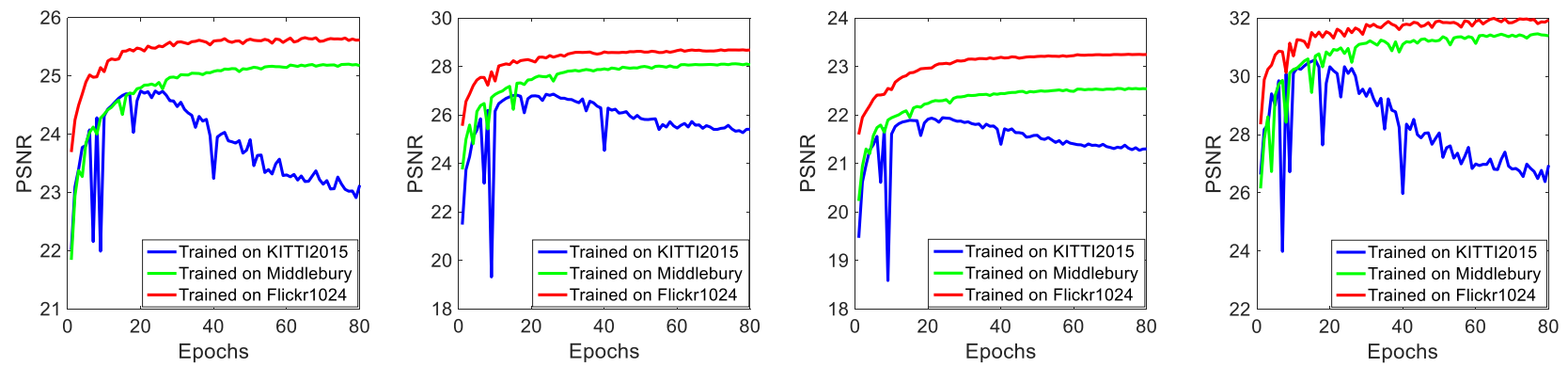

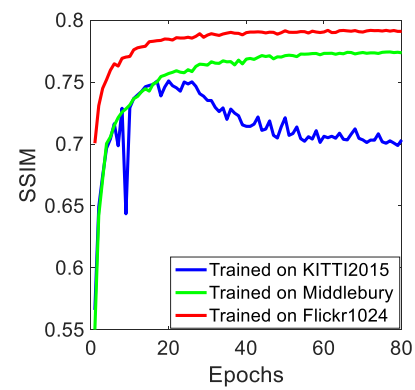

(a) KITTI2015

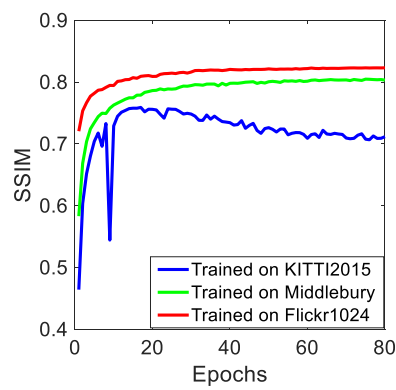

(b) Middlebury

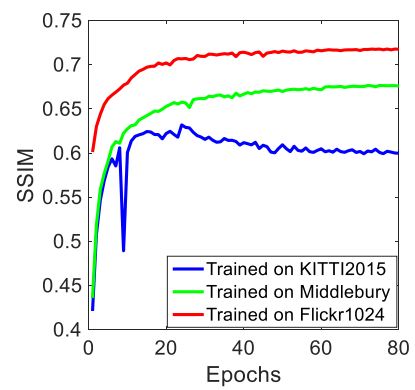

(c) Flickr1024

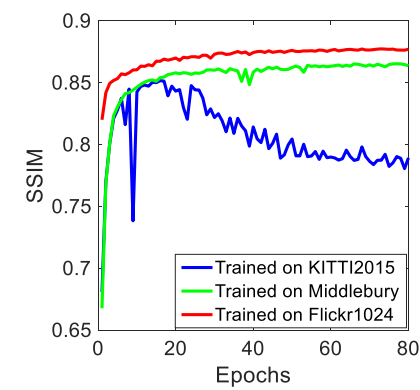

(d) $E T H 3 D$

Figure 4: PSNR and SSIM values achieved by PASSRnet [17] with different numbers of training epochs for $4 \times$ SR. Note that, the performance is evaluated on the test sets of (a) KITTI2015, (b) Middlebury, (c) Flickr1024, and (d) ETH3D, respectively.

\section{References}

[1] E. Agustsson and R. Timofte. Ntire 2017 challenge on single image super-resolution: Dataset and study. In The IEEE Conference on Computer Vision and Pattern Recognition (CVPR) Workshops, volume 3, page 2, 2017. 1, 3

[2] A. V. Bhavsar and A. Rajagopalan. Resolution enhancement in multi-image stereo. IEEE transactions on Pattern Analysis and Machine Intelligence, 32(9):1721-1728, 2010. 1

[3] X. Chen, Q. Zhang, M. Lin, G. Yang, and C. He. Noreference color image quality assessment: From entropy to perceptual quality. arXiv preprint arXiv:1812.10695, 2018. 3

[4] A. Geiger, P. Lenz, and R. Urtasun. Are we ready for autonomous driving? the kitti vision benchmark suite. In Proceedings of the IEEE Conference on Computer Vision and Pattern Recognition (CVPR), 2012. 1, 2, 3

[5] H. Hirschmuller and D. Scharstein. Evaluation of cost functions for stereo matching. In Proceedings of the IEEE Conference on Computer Vision and Pattern Recognition (CVPR), 2007. 1, 2, 3

[6] D. S. Jeon, S.-H. Baek, I. Choi, and M. H. Kim. Enhancing the spatial resolution of stereo images using a parallax prior. In Proceedings of the IEEE Conference on Computer Vision and Pattern Recognition (CVPR), pages 1721-1730, 2018. 1,4

[7] X. Li, H. Huang, H. Zhao, Y. Wang, and M. Hu. Learning a convolutional neural network for propagation-based stereo image segmentation. The Visual Computer, pages 114, 2018. 1
[8] Z. Liang, Y. Feng, Y. Guo, H. Liu, W. Chen, L. Qiao, L. Zhou, and J. Zhang. Learning for disparity estimation through feature constancy. In Proceedings of the IEEE Conference on Computer Vision and Pattern Recognition (CVPR), pages 2811-2820, 2018. 1

[9] C. Ma, C.-Y. Yang, X. Yang, and M.-H. Yang. Learning a no-reference quality metric for single-image superresolution. Computer Vision and Image Understanding, 158:1-16, 2017. 3

[10] M. Menze and A. Geiger. Object scene flow for autonomous vehicles. In Proceedings of the IEEE Conference on Computer Vision and Pattern Recognition (CVPR), pages 30613070, 2015. 1, 2, 3

[11] A. Mittal, A. K. Moorthy, and A. C. Bovik. No-reference image quality assessment in the spatial domain. IEEE Transactions on Image Processing, 21(12):4695-4708, 2012. 3

[12] D. Scharstein, H. Hirschmüller, Y. Kitajima, G. Krathwohl, N. Nešić, X. Wang, and P. Westling. High-resolution stereo datasets with subpixel-accurate ground truth. In German Conference on Pattern Recognition, pages 31-42. Springer, 2014. 1, 2, 3

[13] D. Scharstein and C. Pal. Learning conditional random fields for stereo. In Proceedings of the IEEE Conference on Computer Vision and Pattern Recognition (CVPR), pages 1-8. IEEE, 2007. 1, 2, 3

[14] D. Scharstein and R. Szeliski. High-accuracy stereo depth maps using structured light. In Proceedings of the IEEE Conference on Computer Vision and Pattern Recognition (CVPR), 2003. 1, 2, 3 
[15] T. Schöps, J. L. Schönberger, S. Galliani, T. Sattler, K. Schindler, M. Pollefeys, and A. Geiger. A multi-view stereo benchmark with high-resolution images and multicamera videos. In Proceedings of the IEEE Conference on Computer Vision and Pattern Recognition (CVPR), volume 2017, 2017. 1, 2, 3

[16] L. Wang, Y. Guo, Z. Lin, X. Deng, and W. An. Learning for video super-resolution through HR optical flow estimation. In Proceedings of the Asian Conference on Computer Vision (ACCV), Perth, Australia, 2018. 1

[17] L. Wang, Y. Wang, Z. Liang, Z. Lin, J. Yang, W. An, and Y. Guo. Learning parallax attention for stereo image superresolution. In Proceedings of the IEEE Conference on Computer Vision and Pattern Recognition (CVPR), June 2019. 1, $2,4,5$

[18] T. Xue, B. Chen, J. Wu, D. Wei, and W. T. Freeman. Video enhancement with task-oriented flow. arXiv preprint arXiv:1711.09078, 2017. 1

[19] S. Zhang, Y. Lin, and H. Sheng. Residual networks for light field image super-resolution. In Proceedings of the IEEE Conference on Computer Vision and Pattern Recognition, pages 11046-11055, 2019. 1 